http://jmscr.igmpublication.org/home/ ISSN (e)-2347-176x ISSN (p) 2455-0450

crossref DOI: https://dx.doi.org/10.18535/jmscr/v7i10.59

Journal Of Medical Science And Clinical Research

\title{
Generalised Fixed Drug Eruption: A Retrospective study from a tertiary care centre in South India
}

\author{
Authors \\ Dr Sandhya S Nair ${ }^{1}$, Dr Smitha Ancy Varghese 2* $^{*}$ \\ ${ }^{1}$ Assistant Professor, Department of Dermatology, Government Medical College Thiruvananthapuram \\ ${ }^{2}$ Assistant Professor, Department of Dermatology, Government Medical College Thiruvananthapuram \\ *Corresponding Author \\ Dr Smitha Ancy Varghese
}

Assistant Professor, Department of Dermatology, Government Medical College Thiruvananthapuram, India

\begin{abstract}
Introduction: Fixed drug Eruption (FDE) can occur as both localised and generalised forms, with the generalised form GFDE often requiring hospitalisation and systemic treatment.

Materials and Methods: A retrospective study of ten years was carried out where the case sheets of patients admitted in dermatology ward of a tertiary care centre with GFDE were reviewed. Clinical data, drug history and blood investigations were recorded. Duration of hospital stay and management given were recorded.

Results: 20 patients were diagnosed with GFDE with 10 patients with generalised non bullous FDE and 10 patients with generalised bullous FDE. Most were in the 50-60 age group. Male to female ratio was 3:2. The systemic associations included fever, eosinophilia, hepatic transaminitis and hyponatremia. Most common drug implicated was paracetamol. All the patients were treated with the withdrawal of the culprit drug and supportive measures while $75 \%$ needed additional administration of systemic steroids.

Conclusion: Though GFDE generally has a favourable outcome, hospitalisation, monitoring for systemic involvement and aggressive management may be required in severe cases.

Keywords: Fixed drug eruption, Generalised bullous fixed drug eruption.
\end{abstract}

\section{Introduction}

Fixed Drug Eruption (FDE) is a well documented cutaneous adverse drug reaction characterised by a single (or a few) erythematous/pigmented macule (s), evolving rapidly into an edematous plaque or, infrequently, vesicles/blisters leaving behind a circumscribed area of pigmentation in most cases that recurs at the same site following the administration of the same offending drug. ${ }^{1}$ Ageneralised type of fixed drug eruption (GFDE) characterised by multiple, sharply defined, deep- red macules and blisters of various sizes, bilaterally often in symmetric distribution occurs abruptly within a few hours to days following exposure to offending drug or sometimes the drug of similar group. ${ }^{2}$ This clinical type of FDE may resemble erythema multiforme or the more sinister Stevens-Johnson syndrome or toxic epidermal necrolysis (SJS/TEN) leading to diagnostic difficulties. ${ }^{3}$ In this study we have included cases of generalised FDE both bullous and non bullous types that had more than $10 \%$ body surface area 
involvement, that were admitted in our tertiary care centre during the study period of ten years.

\section{Materials and Methods}

This was a retrospective observational study approved by the Institutional Ethics committee (IEC No. 05/25/2014/MCT) of our institution and was conducted according to the declaration of Helsinki. Data collected from inpatient case records from January 2005 to December 2014 were retrieved from the medical records library of our institution. A total of 506 case sheets pertaining to cutaneous adverse drug reactions were utilised to procure the requisite data. Among them, patients admitted with the diagnosis of GFDE were included in the study. The data collection form was customised to acquire data regarding ADRs. A detailed proforma containing patients' details including demographic data, history of previous drug allergies, clinical history, past history and co-morbidities were recorded. In order to identify the culprit drug, the factors like drug history, temporal correlation with the drug, history of previous drug reactions occurring on the same site, duration of skin lesions, time interval between the drug intake and onset of rash, morphology of drug eruption, associated mucosal or systemic involvement and improvement of lesions on withdrawal of drug were carefully analysed. The routine investigations like complete blood count and urine analysis, liver function test, serum electrolyte levels, renal function test, random blood sugar and other relevant investigations were carried out.

\section{Results}

A total of 20 patients, twelve $(60 \%)$ males and eight $(40 \%)$ females, were recruited in this study. The ages of our patients ranged from 16-88 years with the fifth decade being the most common age group affected with six patients (30\%). When the temporal association with the drug intake and onset of symptoms were considered, four patients had a very short period of up to 8 hours, twelve patients had noticed clinical lesions in a period between 8 and 24 hours and the remaining four patients took more than 24 hours i.e. 2-3 days to notice lesions.

All our patients were diagnosed by their typical clinical manifestation not necessitating a histological confirmation. In cases where intact bulla were present a Tzanck smear showed mostly neutrophils, secondly eosinophils and some cases a combination of both. Fourteen patients (70\%) reported previous similar episodes in the past, with six $(30 \%)$ of them having the same offending drug and the remaining eight patients (40\%) not aware of the previous culprit drug. An unrelated drug was implicated in one case. Six patients $(30 \%)$ were having their first episode with no history of previous drug reaction. On taking a drug history, multidrug consumption was reported in eleven $(55 \%)$ patients, but nine $(45 \%)$ of them had mono drug intake. While identifying the offending drugs was possible in nine $(45 \%)$ cases, it was not distinguishable in eleven (55\%) patients.

Commonly involved sites included trunk, mucosa and upper limbs. Cutaneous involvement without mucosal involvement was observed in six cases $(30 \%)$ and additional oral mucosa involvement in fourteen $(70 \%)$ cases. Genital skin was involved in eleven cases $(55 \%)$. As for the morphology of skin lesions, ten patients $(50 \%)$ had plaque skin lesions, while bullous lesions were found in ten cases $(50 \%)$.

Three patients had constitutional symptoms like fever during the course of hospital stay, four patients developed elevation of transaminases, two patients had peripheral blood eosinophilia and one patient had hyponatremia.

The category of causative drugs included eleven $(55 \%)$ analgesics, three $(15 \%)$ antibiotics and one (5\%) miscellaneous cases. The remaining five cases had been given an analgesic and antibiotic and we were not able to determine the exact causative agent. The most common offending drugs were paracetamol in six $(30 \%)$ cases, diclofenac in three $(15 \%)$ cases, cotrimoxazolein two $(10 \%)$ cases, and, piroxicam and doxycycline 
in one $(5 \%)$ case each. In one case no history of intake of allopathic drugs was elicited instead the patient developed characteristic GFDE within hours of consuming an Ayurvedic drug. Other suspect drugs included pantoprazole, dicyclomine, nimesulide and chloroxazone.

The duration of hospital stay ranged from 2-11 days with a mean duration of 5.7 days. Five patients $(25 \%)$ required only supportive care while the remaining fifteen patients $(75 \%)$ required management with systemic steroids for an average of 4.8 days. All patients recovered with no mortality.

\section{Discussion}

FDE is a common type of drug eruption whose incidence has shown an increasing trend in the recent years ${ }^{4}$. Since most types of FDE involve only a single or a few lesions they do not require admission and an outpatient management is sufficient. However in cases of GFDE, a clinical variant of FDE, the presentation consists of numerous multifocal lesions that clinically resembles erythema multiforme, SJS or TEN and often necessitates an inpatient care. In our study for a period of ten years, twenty such patients were admitted with involvement of skin of more than 10 percent body surface area.

Skin lesions usually manifests as well-defined erythematous to violaceous round or oval plaques as generalized nonbullous fixed drug eruption and is occasionally vesicular or bullous in the case of generalized bullous fixed drug eruption. ${ }^{5}$ In our series we had ten patients who had a non bullous presentation and an equal number of patients with the bullous variant.

The general time of onset of FDE following culprit drug intake ranges from0.5 to 8 hours with a mean time of 2 hours ${ }^{6}$. In our study, majority of our patients $(60 \%)$ noticed lesions between 8-24 hours while $20 \%$ patients noticed only 2-3 days after starting the offending drug.

In other studies of generalised FDE the initial lesions are often seen on lips or genitalia, followed by multiple round to oval well- marginated erythematous edematous plaques or blisters over the rest of theskin. ${ }^{1,7}$ Also it was seen that mucous membrane involvement is more common in the bullous variant than in nonbullous variant of GFDE. ${ }^{7}$ In this study also fourteen (70\%) patients had mucous membrane involvement initially with oral cavity being involved in all the fourteen and additional involvement of genitalia in eleven patients.

Although diagnosis of FDE is easy for dermatologists, a tzanck smear (in case of bullous variant) and histology may contribute diagnostic clues. A tzanck smear in bullous FDE generally shows inflammatory cells with occasional dyskeratotic and acantholytic cells. ${ }^{8}$ In our study group tzanck smear in bullous cases of FDE revealed only inflammatory cells. No dyskerototic cells or acantholytic cells were seen. Histologically FDE is characterised bydyskeratotic eosinophilic cells in the upper epidermis and hydropic degeneration of the basal cell layer resulting in pigmentary incontinence. Marked edema, vascular dilatation, and a perivascular inflammatory infiltrate com- posed of lymphocytes, neutrophils, histiocytes, and mast cells may be conspicuous features in the upper dermis. ${ }^{9}$

Systemic symptoms like fever and complications like elevated liver enzymes and hyponatremia were observed in few of our patients. The prognosis and course of the disease are presumed to be more favourable in GFDE than SJS/TEN. Our patients required only a short hospital stay and all of them were relieved of symptoms at the time of discharge. $75 \%$ patients required a short course of systemic steroids while $25 \%$ patients recovered with only supportive treatment. The current line of management of FDE includes immediate discontinuation of the offending drug, topical antibiotics, topical steroids, emollients and analgesics for symptomatic management. Currently there is no specific therapy targeted to the treatment of GFDE. Systemic treatment with corticosteroids and intravenous immunoglobulins is most often used 
with good results. ${ }^{10}$ Some case reports also suggest cyclosporin to be a favoured treatment option in severe cases. ${ }^{10,11}$

However, in a study comparing prognosis of 58 patients with GBFDE and 170 patients with SJS or SJS / TEN overlap, they were unable to confirm that GBFDE had better prognosis than SJS or SJS/TEN of similar disease extent in older patients and it was concluded that severe cases of GBFDE deserve the attention and active management given to patients with SJS or TEN. ${ }^{12}$ In a study by Gupta et al, cotrimoxazole was the most common cause of the FDE. Other drugs found to cause FDE include oxyphenbutazone, metamezole, tetracycline and piroxicam. ${ }^{11}$ In our series, paracetamol was the most common causative drug, followed by diclofenac, cotrimoxazole, piroxicam and doxycycline. It has been reported that the incidence of paracetamolinduced FDE was $1.5 \%-7.9 \%$ among cases of FDE.$^{13}$ It was also seen that the most common drug causing GBFDE in our series was paracetamol followed by diclofenac. In other case reports, drugs like cotrimoxazole, doxycycline, ciprofloxacin and multiple NSAIDS have been implicated. ${ }^{14} \mathrm{We}$ also had a patient with an Ayurvedic drug as the causative agent. Use of herbal medications and substances are gradually emerging as important culprits in adverse cutaneous drug eruptions and inflammatory reactions. A study by Ayan wolo revealed FDE occurring with the use of a herbal toothpaste in several patients. ${ }^{15}$

\section{Conclusion}

With the introduction of newer analgesics, antibiotics and antiepileptics the incidence scenario of FDE may change in future. Herbal formulations, though widely believed to be safe may be potential culprits for several drug reactions including FDE. Though GFDE has a better prognosis compared to severe cutaneous adverse reactions and management involves symptomatic therapy with antihistamines and topical steroids, severe cases of GFDE can result in systemic complications and may require the aggressive management of TEN.

\section{Sources of support in the form of grants: Nil}

\section{References}

1. Sehgal VN, Srivastava G. Fixed drug eruption (FDE): changing scenario of incriminating drugs. Int J Dermatol. 2006 Aug;45(8):897-908.

2. Daulatabadkar B, Pande S, Borkar M. Generalized bullous fixed drug reaction: A close similarity to stevens-johnson syndrome. Indian $\mathbf{J}$ Drugs Dermatol 2017;3:28-31.

3. Rai R, Jain R, Kaur I, Kumar B. Multifocal bullous fixed drug eruption mimicking Stevens-Johnson syndrome. Indian J Dermatol Venereol Leprol 2002;68:175-6.

4. Lee AY. Fixed drug eruptions. Incidence, recognition, and avoidance. Am J Clin Dermatol. 2000;1:277-285.

5. Jung JW, Cho SH, Kim KH, Min KU, Kang HR. Clinical features of fixed drug eruption at a tertiary hospital in Korea. Allergy Asthma Immunol Res. 2014;6(5):415-420.

6. Sehgal VN, Gangwani OP. Fixed drug eruption. Current concepts. Int J Dermatol. 1987;26:67-74.

7. Mitre, V., Applebaum, D. S, Albahrani, Y., \& Hsu, S. (2017). Generalized bullous fixed drug eruption imitating toxic epidermal necrolysis: a case report and literature review. Dermatology Online Journal, 23(7).

8. Panwar H, Joshi D, Goel G, Asati D, Majumdar K, Kapoor N. Diagnostic utility and pitfalls of Tzanck smear cytology in diagnosis of various cutaneous lesions. J Cytol 2017;34:179-82.

9. Lever WF, Schamberg-Lever G. Fixed drug eruption. In: Lever WF, Schamberg- 
Lever G, eds. Histopathology of the Skin, 8th edn. Philadelphia: Lippincott, 2001.

10. Malviya N, Cyrus N, Vandergriff $T$, Mauskar M. Generalized bullous fixed drug eruption treated with cyclosporine. Dermatol Online J 2017;23.

11. Beniwal R, Gupta LK, Khare AK, Mittal A, Mehta S, Balai M. Cyclosporine in generalized bullous-fixed drug eruption. Indian J Dermatol 2018;63:432-3.

12. Lipowicz $S$, Sekula $P$, Ingen-Housz-Oro $S$, Liss Y, Sassolas B, Dunant A, et al. Prognosis of generalized bullous fixed drug eruption: Comparison with StevensJohnson syndrome and toxic epidermal necrolysis. Br J Dermatol 2013;168:72632.

13. Gupta R. Drugs causing fixed drug eruptions: Confirmed by provocation tests. Indian J Dermatol Venereol Leprol 2003;69:120-1.

14. Girisha BS, Noronha TM, Alva AC, Menon A. Generalized bullous fixed drug eruption mimicking toxic epidermal necrolysis caused by paracetamol. Clin Dermatol Rev 2018;2:34-7.

15. Ayanlowo OO. Fixed drug eruption at a dermatology clinic in Lagos, Nigeria. J Clin Sci 2015;12:108-12. 